\title{
Cryogenic system for COMET experiment at J-PARC
}

\section{Taekyung Ki (Corresponding author)}

Applied Research Laboratory

KEK (High Energy Accelerator Research Organization)

1-1 Oho, Tsukuba, Ibaraki, 305-0801, Japan

Fax : +81-29-864-3209, E-mail address: taekyung@post.kek.jp 


\section{Cryogenic system for COMET experiment at J-PARC}

Taekyung $\mathrm{Ki}^{\mathrm{a}}$, Makoto Yoshida ${ }^{\mathrm{b}}$, Ye Yang ${ }^{\mathrm{a}, \mathrm{c}}$, Toru Ogitsu ${ }^{\mathrm{a}}$, Masami Iio ${ }^{\mathrm{a}}$, Yasuhiro Makida $^{\mathrm{b}}$, Takahiro Okamura ${ }^{\mathrm{b}}$, Satoshi Mihara ${ }^{\mathrm{b}}$, Tatsushi Nakamoto ${ }^{\mathrm{a}}$, Michinaka Sugano ${ }^{\mathrm{a}}$, and Ken-ichi Sasaki ${ }^{\mathrm{a}}$

${ }^{\mathrm{a}}$ Applied Research Laboratory

KEK (High Energy Accelerator Research Organization)

1-1 Oho, Tsukuba, Ibaraki, 305-0801, Japan

${ }^{\mathrm{b}}$ Institute of Particle and Nuclear Studies

KEK (High Energy Accelerator Research Organization)

1-1 Oho, Tsukuba, Ibaraki, 305-0801, Japan

${ }^{c}$ Department of Applied Quantum Physics and Nuclear Engineering

Kyushu University

744 Motooka, Nishi-Ku, Fukuoka 819-0395, Japan 


\section{Abstract}

Superconducting conductors and cryogenic refrigeration are key factors in the accelerator science because they enable the production of magnets needed to control and detect the particles under study. In Japan, a system for COMET (Coherent Muon to Electron Transition), which will produce muon beam lines, is under the construction at J-PARC (Japan Proton Accelerator Research Complex). The system consists of three superconducting magnets; the first is a pion-capture solenoid, the second is a muontransport solenoid, and the third is a detector solenoid. It is necessary to cool down the magnets efficiently using two-phase helium and maintain them securely at $4.5 \mathrm{~K}$. For stable cryogenic refrigeration of the magnets, a suitable cooling method, structures, and the irradiation effect on materials should be investigated. In this paper, we focus on the development of an overall cryogenic system for cooling the capture and transport solenoids. A conduction-cooling method is considered for cooling the capture and transport solenoids because of the advantages such as the reduction of total heat load, fewer components, and simplified structure. To supply cryogenic fluids ( $4.5 \mathrm{~K}$ liquid helium and $58 \mathrm{~K}$ gas helium) and currents to the conduction-cooled magnets subjected to high irradiation, cryogenic components (cooling paths in the magnets, transfer tubes, and a current lead box) are developed. Based on the environment of high irradiation, the conditions (temperature and pressure) of helium in cooling paths are estimated, as well as the temperature of the capture magnet. We develop a dynamic model for quench simulation and estimate the maximum pressure in the cooling pipe when the capture magnet quenches. We conclude with a discussion of the next steps and estimated challenges for the cryogenic system. 
Keywords: Coherent Muon to Electron Transition, cryogenic system, superconducting magnet, irradiation

\section{Introduction}

The muon to electron conversion is one process that involves a charged lepton flavor violation (CLFV). It can be an important clue for new physics beyond the Standard Model $[1,2]$, but has not been observed yet. To overcome the present limit of the branching ratio $\left(7 \times 10^{-13}\right)$, higher muon beam intensity and better beam quality are required. At J-PARC E21, the Coherent Muon to Electron Transition (COMET) experiment is being prepared, and it aims for obtaining an experimental sensitivity of $2.6 \times 10^{-17}$. To satisfy the requirement, a high-intensity muon beam of $10^{11} \mu^{-} / \mathrm{sec}$ with low momentum and a running time of $2 \times 10^{7}$ s are necessary. An $8 \mathrm{GeV}$ proton beam $\left(4.4 \times 10^{13}\right.$ pps intensity) of J-PARC will be used.

Pions are generated by proton bombardment of a target, and muons are produced by pion decays. To achieve a high-intensity muon beam on a stopping target, it is necessary to collect and transport negative pions efficiently. One method is to use superconducting 
solenoid magnets. In a pion-capture solenoid, negative pions with low momentum are captured. In a muon-transport solenoid, the pions decay into negative muons and the muons with low momentum are steered onto a stopping target. In the stopping-target, electron-transport, and detector superconducting solenoid magnets, the negative muons are converted into the monochromatic signal electrons and the electrons are identified [1, 2]. The scheme is well-known, but to achieve the high-intensity muon beam and sensitivity, there are severe requirements such as a high magnetic field for the capture magnet, precise control of magnetic fields, stable cryogenic cooling, and estimation of pressures and temperatures of systems (cooling system and magnets) when a magnet quenches. Furthermore, it is necessary to consider the effect of a high irradiation condition on the systems $[3,4]$.

In this paper, the overall cryogenic system with magnets (capture and transport magnets) for the COMET experiment is described. First, requirements for the magnets and current status are summarized. Second, components of the cryogenic system are designed and the estimated operating conditions are evaluated. Third, when the capture solenoid 1 (CS1) of the capture magnet quenches, the maximum pressure of the cooling pipe is estimated by using dynamic simulation. 


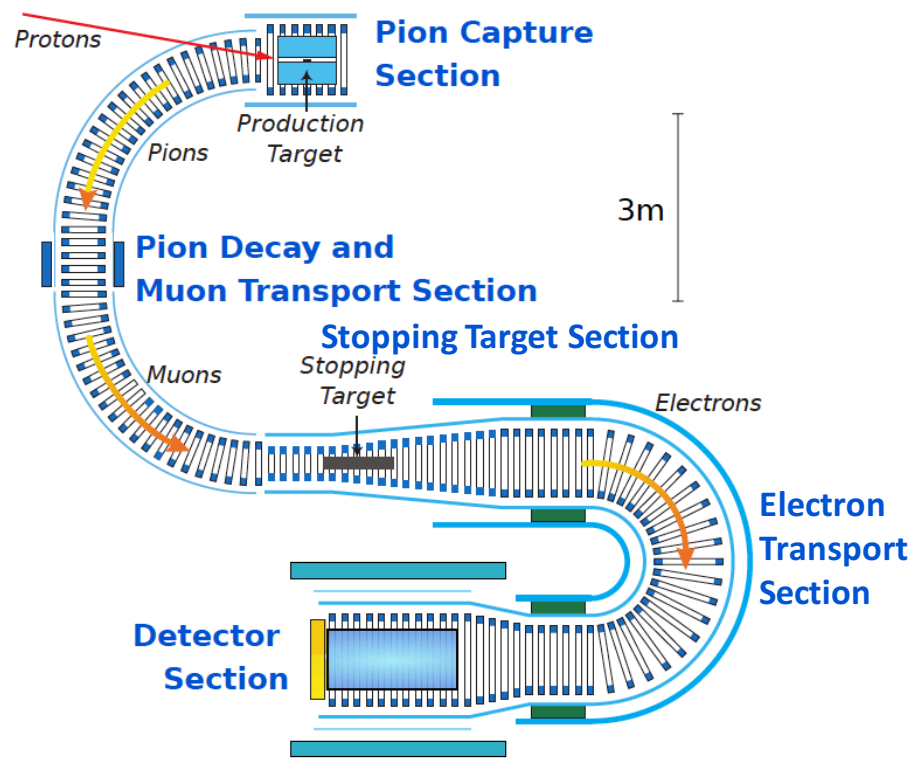

(a) Layout of beam line

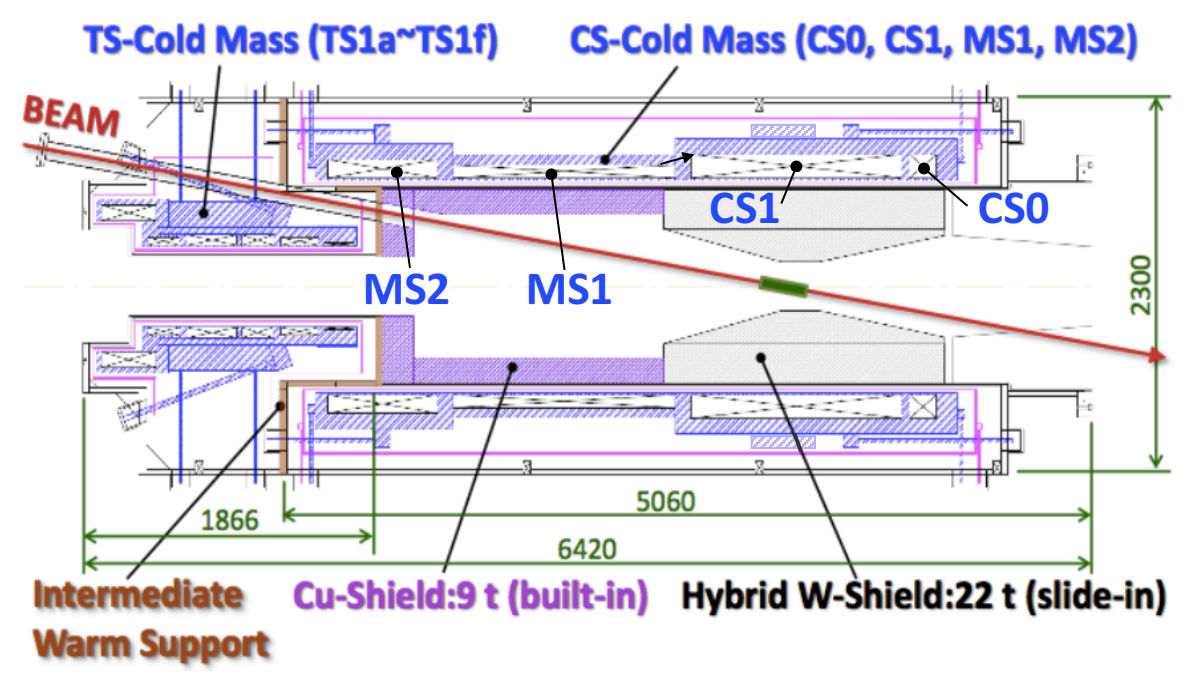

(b) Layout of pion-capture magnet

Fig. 1. Layout of beam line and pion-capture magnet for COMET experiment

(CS: Capture Solenoid, MS: Matching Solenoid, TS: Transport Solenoid). 


\section{Superconducting solenoid magnets}

To acquire low momentum muons efficiently and sufficiently, superconducting magnets are required for the COMET experiment. Such magnets have the advantages of 1) high field with limited space and required configurations to trap pions, 2) smooth change of magnetic field not to trap high momentum particles, and 3) suitable magnetic field to transport low momentum pions and muons. Fig. 1 shows the layout of the beam line and pion-capture magnet for the COMET experiment [3]. There are five magnets; a pioncapture magnet, a muon-transport magnet, a stopping-target magnet, an electron-transport magnet, and a detector magnet. We are presently developing the pion-capture magnet and the muon-transport magnet. It is necessary to check estimated problems prior to development of the magnets. Even though radiation shields are installed around a target (neutrons penetrate thick radiation shields), the capture magnet will be in a high irradiation condition (peak dose: $0.8 \mathrm{MGy} /$ overall operation time). The radiation from neutrons degrades the thermal properties of materials, especially the thermal conductivity, at cryogenic temperatures [5]. And neutrons can make radioactive tritium from liquid helium that cools down the magnet. Therefore, we must consider the potential problems for designing the capture magnet and the cryogenic cooling system. We are continuously developing and modifying the designs of the magnets and cooling system. These issues 
are explained in following sections.

\subsection{Phase I and Phase II}

There are two phases for the COMET experiment. Phase I concerns measuring the level of background and the coherent transition of a muon to an electron with a signal sensitivity of $3.1 \times 10^{-15}$. Any system-level problems identified in phase will be addressed before phase II. For the phase I operation, the whole system consists of the capture, 90 degree-curved transport, and detector magnets. At the end of phase I, the transport magnet will be extended for the 180 degree-curved shape and a detector system with a C-shaped electron-transport magnet will be installed. In phase II, we will measure the coherent transition of a muon to an electron with a signal sensitivity of $2.6 \times 10^{-17}$. Table 1 shows operating conditions of the proton beam and requirements of the system at phase I and phase II.

\section{Table 1}

Operating conditions of proton beam and requirements of system at phase I and phase II.

\begin{tabular}{lllll}
\hline Experiment & Sensitivity & $\begin{array}{l}\text { Stopped muon } \\
\left(\mu^{-} / \mathrm{s}\right)\end{array}$ & $\begin{array}{l}\text { Beam intensity } \\
(\mathrm{pps})\end{array}$ & $\begin{array}{l}\text { Beam power } \\
(\mathrm{kW})\end{array}$ \\
\hline Phase I & $3.1 \times 10^{-15}$ & $1.3 \times 10^{9}$ & $2.5 \times 10^{12}$ & 3 \\
Phase II & $2.6 \times 10^{-17}$ & $10^{11}$ & $4.4 \times 10^{13}$ & 56 \\
\hline
\end{tabular}




\subsection{Required magnetic condition}

Fig. 2 shows the required profile of the magnetic flux density for the COMET experiment of phase II. At the axis of the capture magnet, the maximum magnetic field is $5 \mathrm{~T}$ to collect as many the low energy pions (transverse momentum below $113 \mathrm{MeV} / \mathrm{c}$ ) as possible $[1,2]$. Backward-scattered pions are efficiently transferred by changing adiabatically the magnetic field from $5 \mathrm{~T}$ to $3 \mathrm{~T}$. In the capture magnet, the smooth decrease of the magnetic field makes the pion beam more parallel. The muon-transport magnet produces $3 \mathrm{~T}$ at the center line to eliminate energetic muons and to transport the muons of low momentum around $40 \mathrm{MeV} / \mathrm{c}$ while the transported pions decay into muons along the magnet of $\sim 12 \mathrm{~m}$ length (12 $\mathrm{m}$ is for phase II and at phase I, the length is $6 \mathrm{~m}$ ). Since the transport magnet is a kind of curved solenoid, it creates a drift of the center of the helical trajectory for a charged particle and the drift depends on the magnet field [1, 2]. By using the drift, we can select the muons with low momentum. To compensate for their drift, dipole fields $(0.03-0.05 \mathrm{~T})$ are applied to the transport magnet. 


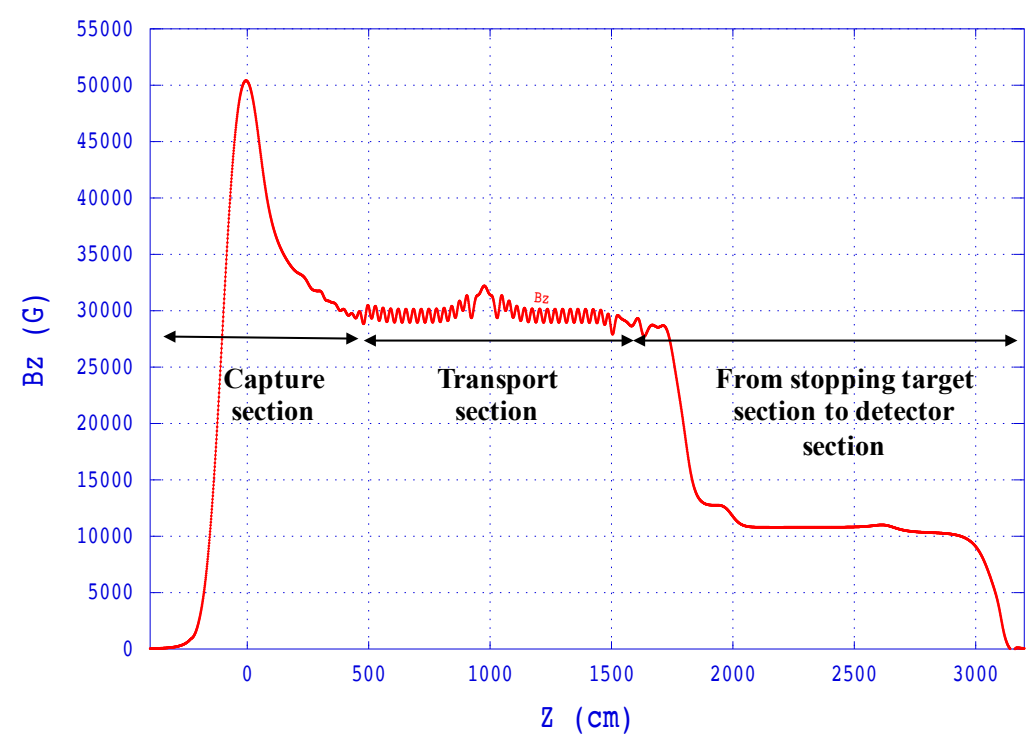

Fig. 2. Distribution of magnetic flux density along beam axis for COME experiment of phase II.

\subsection{Pion-capture magnet}

The capture magnet uses an aluminum-stabilized superconductor to reduce the heat generated from radiation. The configuration and parameters of the capture magnet were already explained in our previous paper [4]. Table 2 shows current parameters with some changes for the magnet. The main issues are 1) the effect of irradiation on materials and 2) designs of cooling paths and support structures. Irradiation degrades the performance of the superconductor, but we believe the effect is very small at our operating condition. On the other hand, the thermal conductivity and electrical resistivity of conductors are severely affected by the irradiation. BT+Epoxy prepreg is used for this magnet because 
of its radiation resistivity [6], but the thermal conductivity of the prepreg is not exactly known around at $4.5 \mathrm{~K}$. We use $0.01 \mathrm{~W} / \mathrm{m}-\mathrm{K}$ as a worst-case value. The thermal conductivity is very important for estimating the normal operating temperature and the maximum temperature of the magnet during quenches. We are therefore in the process of measuring the thermal conductivity of the prepreg in order to calculate the temperatures. Table 3 shows estimated magnetic forces and the maximum stress on the coils of this magnet. Eight axial and radial supports connect the sections (CS0, CS1, MS1, and MS2) of magnet to the cryostat. Eight supports connect TS1 to the cryostat. The supports are designed to keep the positon of magnet stable. Ribs are added on the shells of magnet and the cryostat to reduce stresses.

\subsection{Muon-transport magnet}

The configuration and parameters for the transport magnet were already explained in our previous paper [4]. Recently, a half (90 degree bending angle for phase I) of the transport magnet are fabricated by TOSHIBA and installed at the hadron hall for the phase I operation. We allow tolerance of $1 \mathrm{~mm}$ for the position of each coil. 
Table 2

Design parameters of capture magnet and heat generation on coils.

\begin{tabular}{ll}
\hline Items & Values \\
\hline Conductor & Aluminum stabilized SC cable \\
& $\mathrm{A} 1 / \mathrm{Cu} / \mathrm{NbTi}=7.3 / 0.9 / 1$ \\
Cable dimensions & $15.3 \times 5.0 \mathrm{~mm}^{2}$ (with insulation) \\
Cable insulation & Polyimide film/Boron-free glass cloth/BT-Epoxy prepreg \\
Magnet length & $\sim 6$ meters \\
Operating current & $2700 \mathrm{~A}$ \\
Max. field on conductor & $5.4 \mathrm{~T}(\mathrm{Tcs} *=6.5 \mathrm{~K})$ \\
Stored energy & $47 \mathrm{MJ}$ \\
Coil inner diameters & $1374 \mathrm{~mm}(\mathrm{CS} 0), 1344 \mathrm{~mm}(\mathrm{CS} 1, \mathrm{MS} 1, \mathrm{MS} 2)$, \\
& $500 \mathrm{~mm}(\mathrm{TS} 1 \mathrm{a}-\mathrm{TS} 1 \mathrm{e}), 820 \mathrm{~mm}(\mathrm{TS} 1 \mathrm{f})$ \\
Coil layers & $9(\mathrm{CS} 0, \mathrm{CS} 1), 5$ (MS1), 7 (MS2), 1 - 6 (TS1a - TS1f) \\
Max. heat generated by & $\mathrm{CS} 0: 0.56 \mathrm{~W}$ and 16 W, CS1: 5 W and 133 W, MS1: 0.44 W and \\
radiation & $56.6 \mathrm{~W}, \mathrm{MS} 2: 0.18 \mathrm{~W}$ and 24.1 W, TS1: 0.03 W and 3.72 W \\
(phase I and phase II) &
\end{tabular}

* Tcs: Current-sharing temperature

\section{Table 3}

Estimated magnetic forces and maximum stress on capture magnet.

\begin{tabular}{llllll}
\hline \multirow{2}{*}{ Sections } & \multicolumn{2}{l}{ All coils : ON } & \multicolumn{2}{l}{ CS and MS : ON, TS: OFF } & Maximum von Mises \\
\cline { 2 - 4 } & $\mathrm{Fr}^{*}(\mathrm{kN})$ & $\mathrm{Fz}^{*}(\mathrm{kN})$ & $\mathrm{Fr}(\mathrm{kN})$ & $\mathrm{Fz}^{*}(\mathrm{kN})$ & stress on coils (MPa) \\
\hline CS0 & 5858 & 6225 & 5858 & 6225 & \\
CS1 & 63527 & 2120 & 63526 & 2120 & 55.2 at CS1 \\
MS1 & 28276 & -3320 & 28272 & -3323 & \\
MS2 & 15972 & -5232 & 15954 & -5253 & \\
TS1 & 8612 & -415 & 8422 & -1016 & \\
\hline
\end{tabular}

* Fr and $\mathrm{Fz}$ mean the force (+ signal: direction from inside to outside) of radial direction and the force $(+$ signal: direction from $\mathrm{CS} 0$ to MS2) of axial direction, respectively. 


\section{Design of cryogenic system}

The capture and transport magnets are cooled by two-phase forced helium flow. We choose the conduction-cooling method which uses cooling pipes and conductors connected with the magnets. Fig. 3 shows the configuration of conduction-cooling for the capture magnet. The method has advantages such as reduction of total heat load, fewer components, small pressure rise (quench case), and simpler structure in comparison with the bath-cooling concept. Additionally, for a magnet in the high irradiation condition, conduction-cooling reduces the effect of radiation on the helium because it reduces the total quantity of liquid and increases the distance between radiation sources and the liquid.

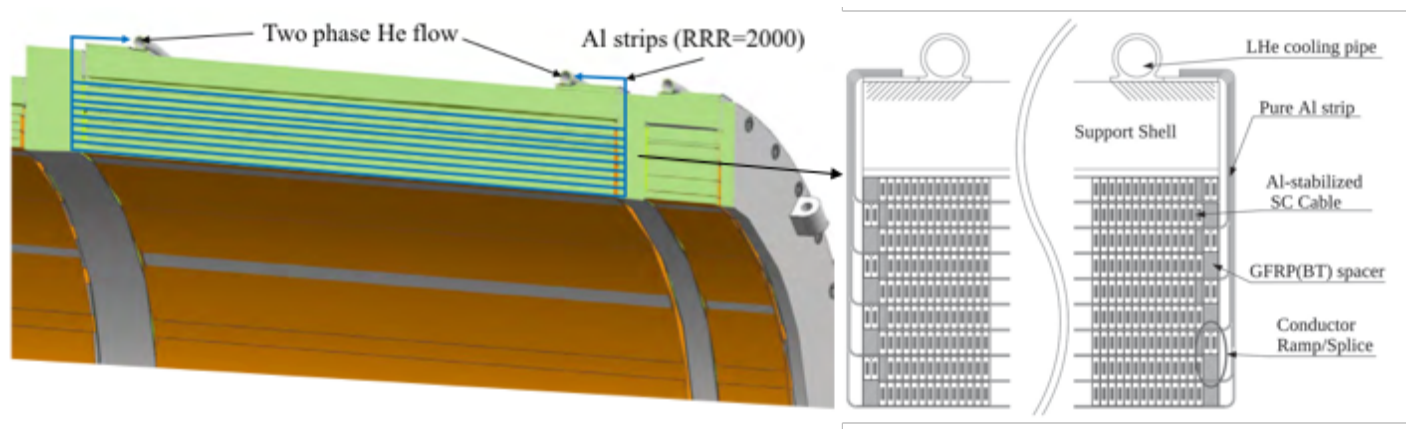

Fig. 3. Configuration of conduction-cooling for capture magnet.

\subsection{Cooling concept for superconducting magnets}

Conduction-cooling is achieved by welding a cooling pipe to the shells of a magnet. The 
shells and the superconductors with stabilizers become thermal paths. For the transport magnet, since the thicknesses of superconducting coils and the intensity of radiation are much smaller than those of capture magnet, additional thermal paths between layers of each coil are not necessary. After making each coil of the transport magnet, two $5 \mathrm{~N}$ (99.999\% purity) aluminum strips are applied to cover the outer surface. Then, a dipole coil is attached on the outer surfaces of the aluminum strips. The aluminum strips are then connected to an omega-type cooling pipe. However, in case of the capture magnet, additional thermal paths are required. The superconducting wire of the capture magnet has the aluminum stabilizer. The number of layers for CS0 and CS1 coils is 9 and the distance between inner and outer radiuses is around $150 \mathrm{~mm}$. To make the magnet thermally stable at the operating condition, it is necessary to design carefully thermal paths and check the effect of thermal paths on the temperature of the magnet.

To do this, we considered the effect of neutrons on the thermal conductivity and the heat deposition from the irradiation in the thermal simulation of CS1 coil [7]. When the neutron influence on CS1 becomes around $6 \times 10^{20} \mathrm{n} / \mathrm{m}^{2}$ (operation of 60 days), the maximum temperature of CS1 approaches the current-sharing temperature of $6.5 \mathrm{~K}$ (after 60 days, we will stop operating the capture magnet, and then increase the temperature of 
the capture magnet to the room temperature to recover the thermal conductivity of aluminum). The thermal conductivity of aluminum $(R R R=2000)$ and deposited heat on CS1 become the thermal conductivity of aluminum $(\mathrm{RRR}=100)$ and $0.03 \mathrm{~W} / \mathrm{kg}$, respectively. The temperature change of CS1 with the current configuration of thermal paths is shown in Fig. 4. Obviously, 5N aluminum strips between layers of the capture magnet make this magnet thermally stable. However, we need to optimize the thicknesses of aluminum strips because the spaces (1. between layers and 2. between coils) for the aluminum strips can decrease the magnetic flux density. Table 4 shows the dimensions of aluminum strips for the CS1 coil.

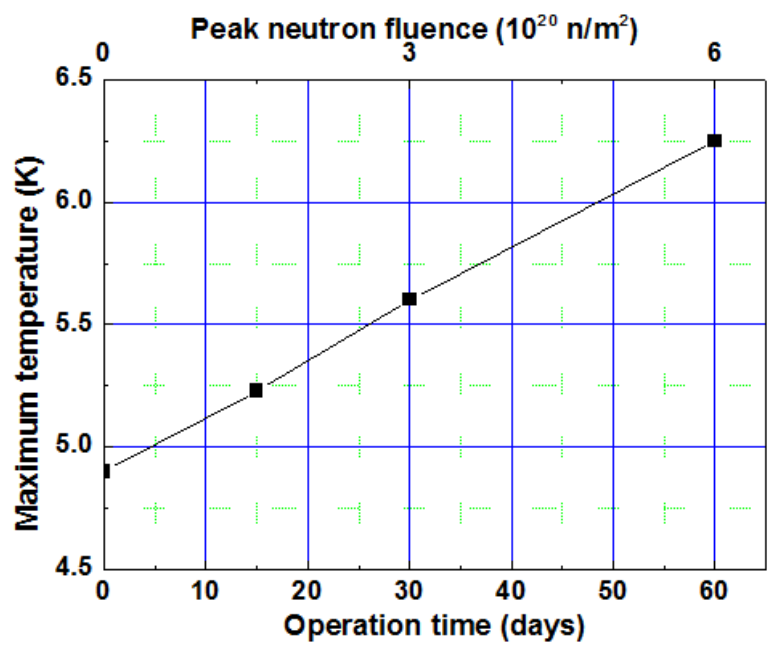

Fig. 4. Temperature change of CS1. 
Table 4

Dimensions of aluminum strips for CS1.

\begin{tabular}{ll}
\hline Al strips & Dimensions \\
\hline Normal Al strips between layers & Thickness : $1 \mathrm{~mm}$, length between cooling pipe and end of each \\
& layer: $5 \mathrm{~cm}-25 \mathrm{~cm}$ \\
Al strip on inner surface of CS1 & Thickness : $3 \mathrm{~mm}$, length between cooling pipe and end of inner \\
& surface: $25 \mathrm{~cm}$ \\
\hline
\end{tabular}

Table 5

Estimated total heat loads at phase I and phase II.

\begin{tabular}{|c|c|c|}
\hline & Phase I & Phase II \\
\hline \multirow{2}{*}{ Thermal radiation } & \multicolumn{2}{|c|}{ Capture: $\sim 5 \mathrm{~W}$} \\
\hline & \multicolumn{2}{|c|}{ Transport: $\sim 2.9 \mathrm{~W}$} \\
\hline Thermal & \multicolumn{2}{|c|}{ Capture: $~ 8.3 \mathrm{~W}$} \\
\hline conduction & \multicolumn{2}{|c|}{ Transport: $\sim 4.2 \mathrm{~W}$} \\
\hline \multirow{2}{*}{ Nuclear heating } & Capture: $6.3 \mathrm{~W}$ & Capture: $234 \mathrm{~W}$ \\
\hline & Transport: $0.02 \mathrm{~W}$ & Transport: $0.6 \mathrm{~W}$ \\
\hline \multicolumn{3}{|l|}{ Heat loads to } \\
\hline CLB, TRT, and & $\sim 40 \mathrm{~W}$ & $\sim 40 \mathrm{~W}$ \\
\hline \multicolumn{3}{|l|}{ bayonets } \\
\hline $\begin{array}{l}\text { Total heat loads at } \\
4.5 \mathrm{~K}\end{array}$ & $\sim 67 \mathrm{~W}$ & $\sim 295 \mathrm{~W}$ \\
\hline Heat loads at & Capture: $\sim 223 \mathrm{~W}$ & Capture: $\sim 283 \mathrm{~W}$ \\
\hline thermal shields & Transport : $\sim 110 \mathrm{~W}$ & Transport : 116 W \\
\hline
\end{tabular}




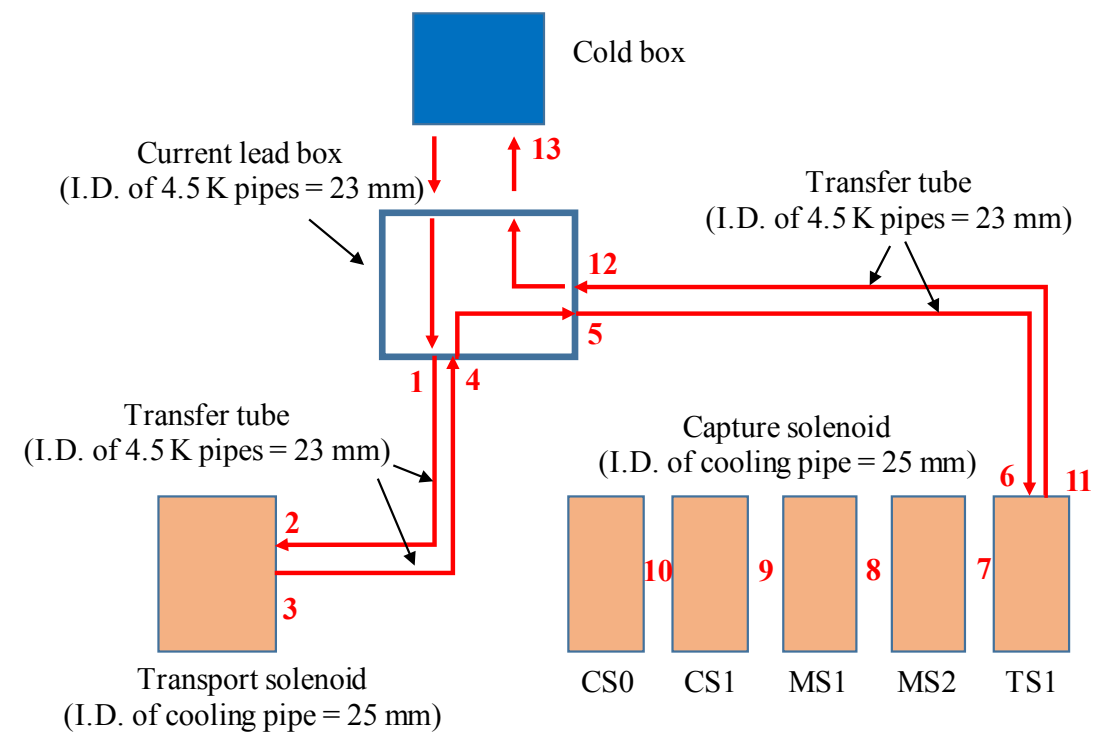

Fig. 5. Flow direction of two-phase forced helium in cryogenic system (I.D. means an inner diameter of pipe).

\subsection{Condition of liquid helium in cooling pipe}

In our cooling concept, two-phase forced helium flow is used. We need to determine required mass flow rates at phase I and phase II and to check flow conditions such as temperature, pressure, and quality. Table 5 shows the estimated total heat loads at phase I and phase II. Fig. 5 shows the flow direction of two-phase helium in the cryogenic system. To keep the two-phase flow stable, the quality of two-phase helium must be less than 0.5 [8]. The total pressure drop in the all pipes which connect a liquefier to magnets must not go beyond an acceptable level. To determine the mass flow rates and the inner diameters of pipes, a model for the 1D nodal analysis is developed by using MATLAB. 
At the research of Haruyama et al., the flow pattern in the cooling pipe was estimated from the Baker diagram of helium as the stratified flow, but experimental results indicated that it behaves as the bubble flow in the pressure drop calculation. Therefore, the homogenous model is used to calculate the pressure drops at this analysis. Pressure drops generated in valves are ignored. Fig. 6 shows the calculated pressure drops, temperatures, and qualities along the pipes. From the results, we confirm that the required mass flow rates at phase I and phase II are $8-10 \mathrm{~g} / \mathrm{s}$ and $32-33 \mathrm{~g} / \mathrm{s}$, respectively. The $25 \mathrm{~mm}$ and $23 \mathrm{~mm}$ of inner diameters are acceptable for the cooling pipes and transfer pipes, respectively. And, flow patterns are estimated as the stratified flow (phase I) or the bubble flow (phase II: from the stratified flow to bubble flow) based on the Baker diagram of helium. The reason why the temperatures decrease along the cooling pipe is that saturation temperatures decrease due to the pressure drops. 

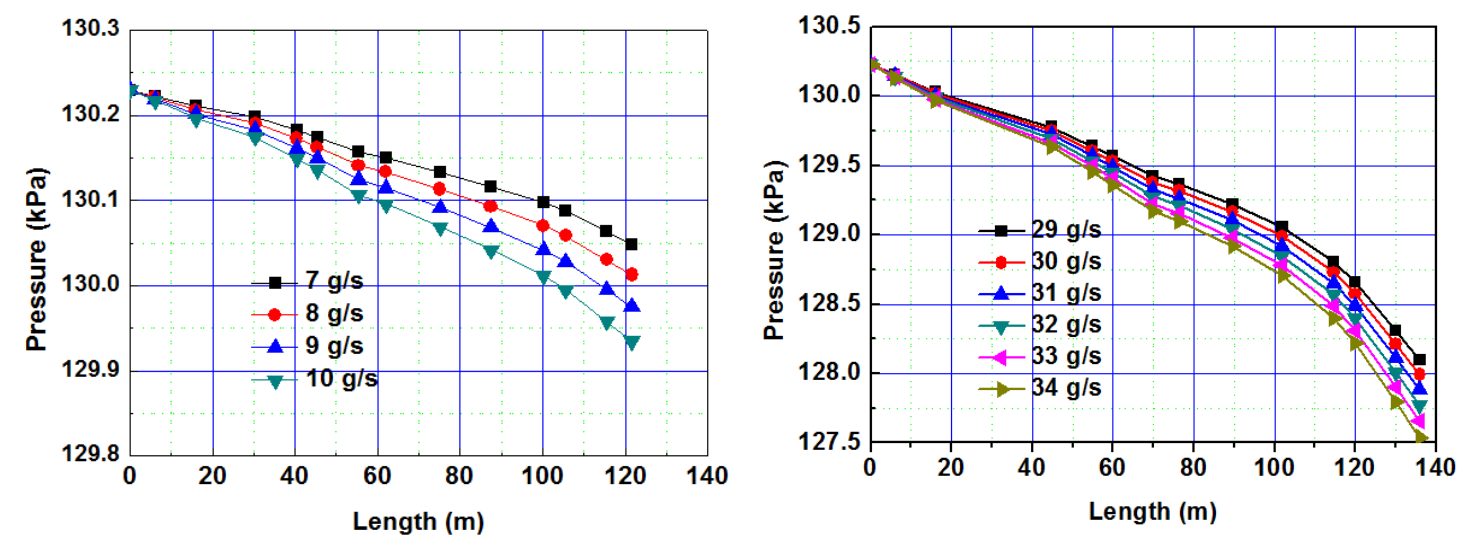

(a) Pressure curves (left: phase I, right: phase II)
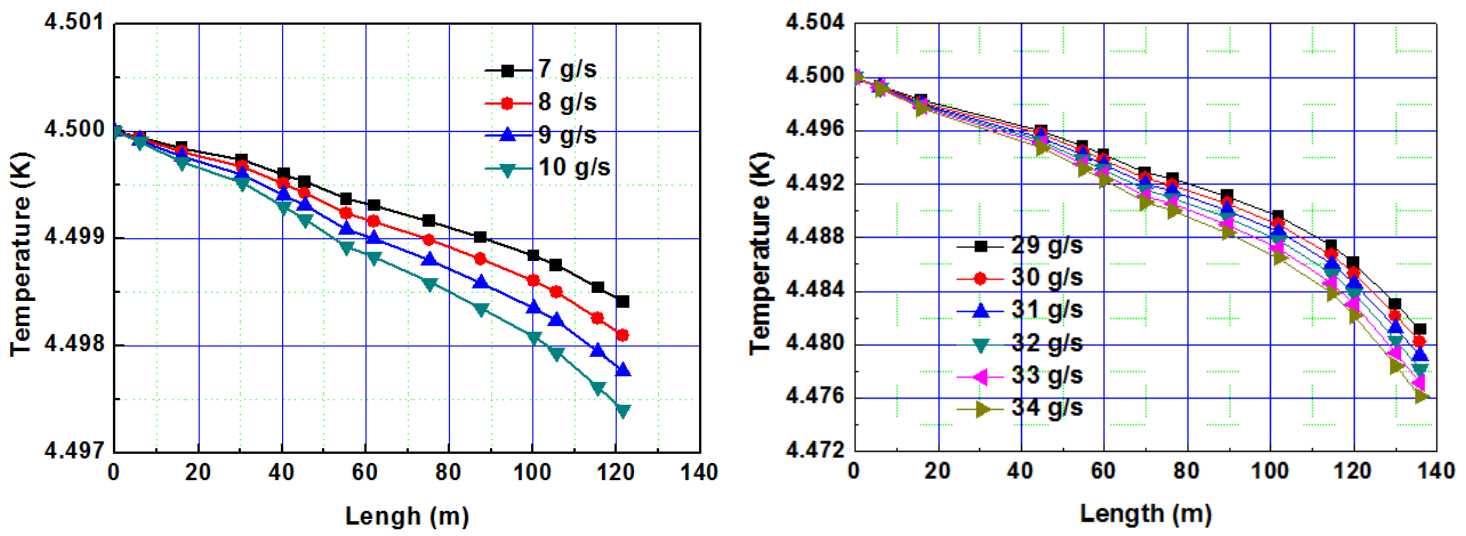

(b) Temperature curves (left: phase I, right: phase II)
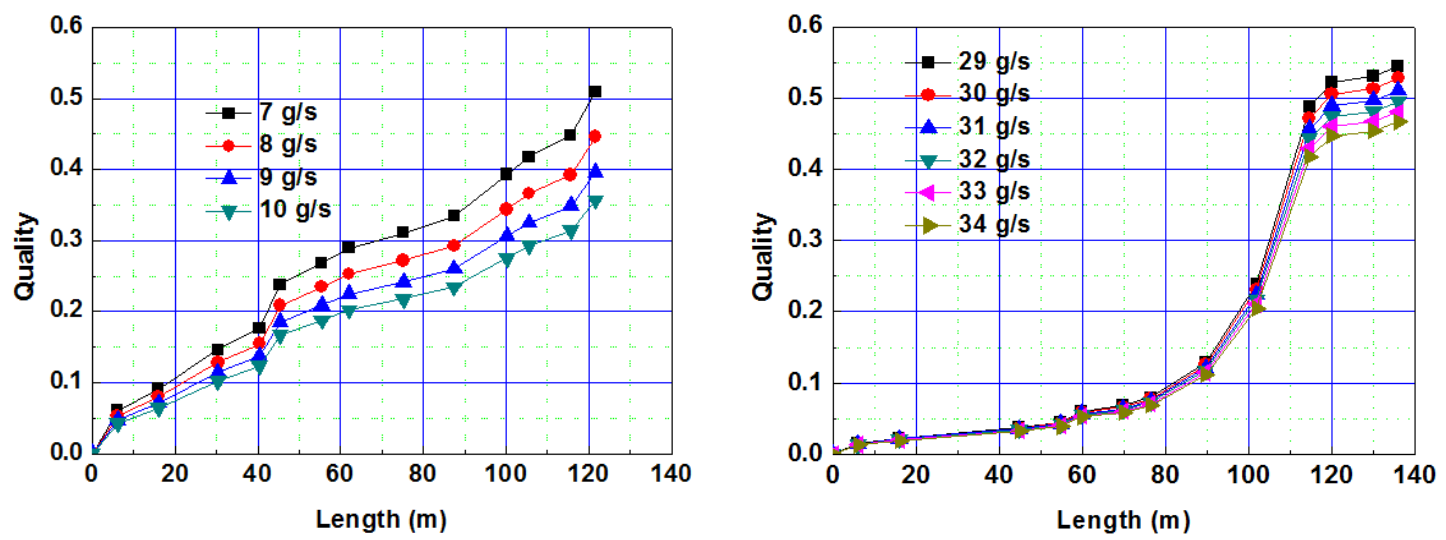

(c) Quality curves (left: phase I, right: phase II)

Fig. 6. Calculated pressure drops, temperatures, and qualities along pipes. 


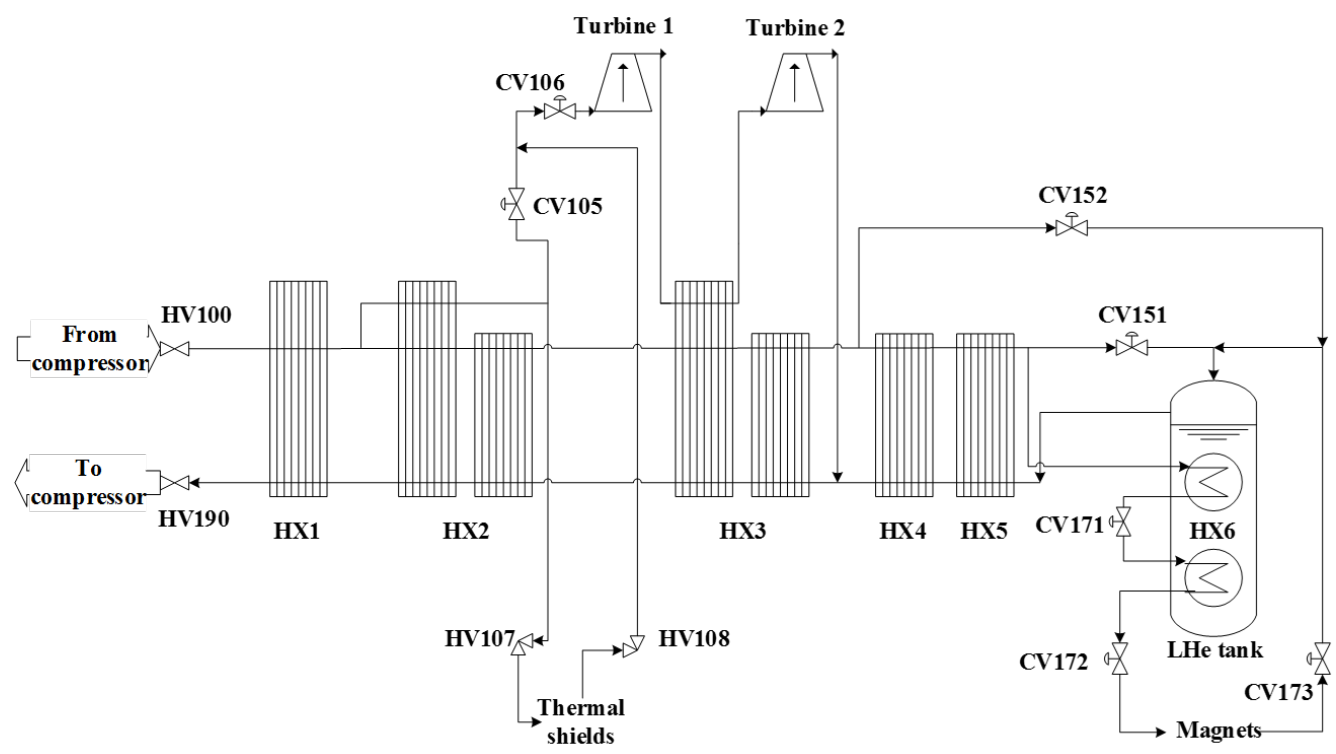

Fig. 7. Overall flow diagram of Linde TCF 50 liquefier.

\subsection{Liquefier}

It is necessary to check that a liquefier can support the required liquid helium flow rate.

The liquefier is a Linde TCF 50 with a cooling capacity of $140 \mathrm{~W}$ at $4.5 \mathrm{~K}$ when it operates in refrigeration mode. Fig. 7 shows an overall flow diagram of the liquefier. The highpressure helium gas exiting from the middle section of HX2 cools down thermal shields of magnets and then goes to turbine 1 . The control valve (CV) 106 maintains an adequate temperature difference (Maximum $\Delta \mathrm{T}=30 \mathrm{~K}$ ) between the LHe and the first coil of a magnet during the cool-down period. To check whether the liquefier can make the mass flow rate of $\sim 10 \mathrm{~g} / \mathrm{s}$ for the application at phase I, we assume that the temperature and mass flow rate of the outlet of high-pressure flow at HX5 are $6.6 \mathrm{~K}$ with $0.9 \mathrm{MPa}$ and 15 
$\mathrm{g} / \mathrm{s}$, respectively. $5 \mathrm{~g} / \mathrm{s}$ goes to the JT valve (CV151) and $10 \mathrm{~g} / \mathrm{s}$ goes to the heat exchanger (HX6) at the inside of LHe tank. The temperature and pressure of liquid helium at the inlet of the magnet system are $4.5 \mathrm{~K}$ and $0.1303 \mathrm{MPa}$. It means that heat of $124 \mathrm{~W}$ is rejected from HX6 and the liquid helium at the inside of the LHe tank absorbs the heat. A required net mass flow rate of liquid helium in the LHe tank is $6.3 \mathrm{~g} / \mathrm{s}$ to absorb the heat completely. The helium flow of $5 \mathrm{~g} / \mathrm{s}$ to the JT valve makes liquid helium flow of 1.6 $\mathrm{g} / \mathrm{s}$ and the return helium flow from the magnet has liquid helium of $5 \mathrm{~g} / \mathrm{s}$ at least. Therefore, the net liquid helium flow to the LHe tank is $6.6 \mathrm{~g} / \mathrm{s}$ and it means that the liquefier can support the COMET experiment for phase I. For phase II, we need to change the liquefier, and the Linde LR280 will be an option (the cooling capacity of a new liquefier should be larger than $600 \mathrm{~W}$ at $4.5 \mathrm{~K}$ ). Our plan is to use the Linde TCF 50 for the experiment of phase I and after finishing the experiment, we will replace the Linde TCF 50 to a new liquefier. Additionally, we need to check cool-down and warm-up time for the whole system because the capture magnet cannot continually be operated due to the degradation of thermal conductivity of the conductor. It means that we will make thermal cycles for the capture magnet to recover the thermal conductivity at room temperature. To estimate whole operation time with the thermal cycles for the whole system, the cool-down and warm-up time needs to be known. A dynamic model for the 
whole system is under study to evaluate the cool-down and warm-up time in simulation.

\subsection{Current lead box}

The current lead box is still being designed. In this paper, we describe the overall concept of the current lead box. Due to the high radiation condition near the magnets, we cannot install the current lead box near the magnets. The cold box and the current lead box will be installed at the first floor level and the magnets will be installed at the underground level. We will install concrete blocks between the first floor and the underground level. The current lead box will contain 10 HTS current leads $(2$ x 3000 A, 1 x 500 A, 3 x 250 A for capture magnet, 1 x 500 A, 3 x 250 A for transport magnet). For the current leads of the transport magnet, the first stage of a two-stage cryocooler (SRDK-408D2, Sumitomo Heavy Industries, Ltd.) cools down copper current leads to about $40 \mathrm{~K}$, and the second stage of the two-stage cryocooler cools down the HTS current leads to about $6 \mathrm{~K}$. HTS tapes are connected with the cold-ends of the HTS current leads and the HTS tapes are thermally anchored on a $4.5 \mathrm{~K}$ return pipe of the transport magnet. For the current leads of the capture magnet, three single-stage cryocoolers $(\mathrm{CH}-110$, Sumitomo Heavy Industries, Ltd.) cool down copper current leads and the warm ends of HTS current leads to about $40 \mathrm{~K}$. HTS tapes are connected with the cold-ends of the HTS current leads 
and the tapes are thermally anchored on a $4.5 \mathrm{~K}$ return pipe of the capture magnet. In the current lead box, the section of the transport magnet is separated from the section of the capture magnet because we will only fabricate the components of the transport magnet at the first time and test the transport magnet. In the meantime, we will fabricate the components for the capture magnet and assembly them with the components of the transport magnet. At joint parts in the current lead box, plates of aluminum nitride are used to make the conditions of good heat transfer and electrical insulation, simultaneously. Flexible bars made of stainless steel are installed together with the HTS current leads to make back-up current paths and protect the HTS current leads when one of magnets quenches.

\subsection{Transfer tube}

Generally, there are two kinds of transfer tubes. The first is a typical and small transfer tube to supply liquid helium for small experiments. The second is a large transfer tube with a thermal shield, supply pipes, return pipes, and bellows for large cryogenic systems. We are developing the stable and efficient large transfer tubes for the magnets. We want to estimate all deformations and stresses of pipes and heat leaks at all operating conditions. We prefer to avoid bellows, in order to simplify the structure and reduce welding. Two 
transfer tubes connect the magnets to the current lead box. The determined locations of magnets and current lead box induce that transfer tubes have complicate shapes and that is why we want to make a simple structure. Once the transfer tubes are installed and we operate the whole system, it will be difficult to fix and modify the transfer tubes because of concrete blocks between magnets and the first floor, and the high radiation condition at the underground level. Therefore, we have to develop an accurate design method and choose the best configuration and structure for the transfer tubes. Furthermore, the transfer tubes include superconducting wires (NbTi) inside. The superconducting wires transfer the currents from the HTS tapes of the current lead box to the first coils of the magnets. Therefore, we need to accurately predict deformations of the inside pipes and keep enough spaces between them at all operating conditions.

Basically, the transfer tubes consist of a vacuum jacket, a thermal shield, two supply pipes of $58 \mathrm{~K}$ and $4.5 \mathrm{~K}$ helium flows, two return pipes of 58 - $62 \mathrm{~K}$ and $4.5 \mathrm{~K}$ helium flows, joint structures for superconducting wires, and spacers. To install superconducting wires at the insides of transfer tubes, thermal joint structures are required and the joints are installed on $4.5 \mathrm{~K}$ return pipes. In the boundary conditions, we developed a design method and procedure for the transfer tubes. Fig. 8(a) and (b) show a developed flow diagram of 
design method and a cross-sectional view of the transfer tube for the transport magnet.

We can calculate all deformations of pipes and stresses on the pipes for the transfer tubes.

But, there is an unknown factor which is about the thermal contact resistance between superconducting wires and the joint structure. The superconducting wires are roundshaped, so the contact conditions between the wires and the surface of the joint are line contacts. Even though we fill out the void volume of the contact spaces with a thermal grease, the contact conditions can make temperature difference between the wires and a 4.5 $\mathrm{K}$ return pipes. Also, the thermal contact resistance determines the length of the joint structure and the gap size between the joints. We are measuring the thermal contact resistance.

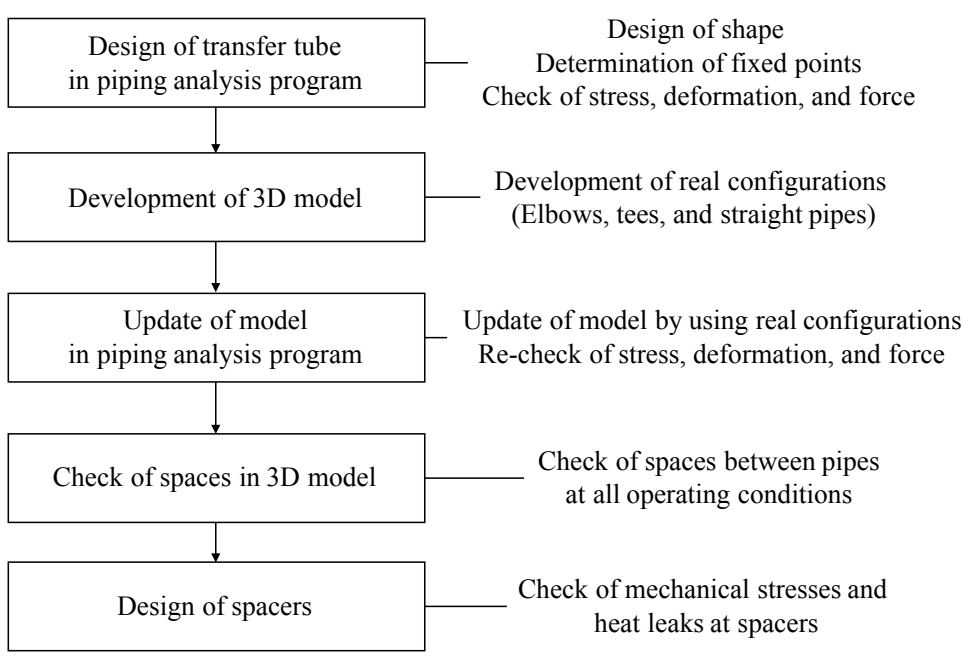

(a) Flow diagram of design method 


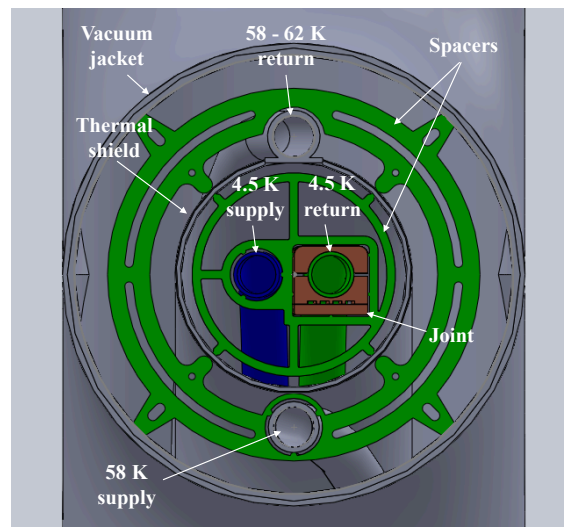

(b) Cross-sectional view

Fig. 8. Developed design method and cross-sectional view of transfer tube.

\section{Dynamic simulation for quench case}

Dynamic simulation is very important to estimate the maximum temperature of magnet and the maximum pressure of $4.5 \mathrm{~K}$ cooling pipe when a magnet quenches. We calculated the maximum temperature of CS1 coil of the capture magnet when the CS1 section quenched [7]. When a magnet quenches, a large amount of heat is transferred to liquid in a $4.5 \mathrm{~K}$ cooling pipe, increasing its temperature and pressure. Generally, the maximum pressure is calculated by using the ratio between densities of liquid and gas phases with a safety factor, assuming relief valves are installed on system of superconducting magnets for safety. But we do not want to install any relief valves near to the magnets because helium is activated by neutron and it is difficult to recover helium vented from a relief valve at the underground level. Our piping systems are designed to sustain pressure of 2 
$\mathrm{MPa}$ and it is the maximum pressure that is allowed for cryogenic piping systems in the hadron hall at J-PARC. It means that we have to accurately estimate the maximum pressure of cooling pipe. By using the results of previous simulation [7], we developed a dynamic model in EcosimPro [9]. Cryogenic components such as pipes, valves, and tanks are developed from the cryogenic library (CRYOLIB 1.1.1) of EcosimPro. Magnets and PID controllers are developed from the THERMAL, PLC, CONTROL libraries of EcosimPro.

\subsection{Dynamic model}

From previous quench analysis [7], we calculate a profile of heat generation for a quench of CS1. The quench of CS1 is considered the worst case in terms of heat generation because CS1 is the longest, and operates in the conditions of the highest irradiation and magnetic flux density. The previous quench analysis used several assumptions. Only CS1 is included, and the outer surfaces of CS1 are at adiabatic conditions. In the real configuration of the capture magnet, the aluminum shells (CS0, CS1, MS1, and MS2) are connected with each other and will transfer heat from the magnet to the cooling pipe. We therefore included a contingency factor for the resistance of coil, which means the heat might be overestimated. The previous quench analysis will be updated to consider the real 
configuration. In CS1, the worst quench position is at the innermost layer of CS1 because of the effect of the high radiation and magnetic field on the electrical resistivity of aluminum stabilizer. The peak heat generation is about $140 \mathrm{~kW}(63 \mathrm{~kW}$ without contingency). The profile of heat generated during the worst quench case is imported to a dynamic model in EcosimPro and it is used for all quench cases. Fig. 9 shows a developed diagram for the dynamic simulation and it is described in EcosimPro. The model consists of a supply section, a magnet section (CS0, CS1, MS1, MS2, and TS1), a return section, a cooling pipe section, a recovery section without a heater, and PID controls with valves. All pipes are 1D-pipe models of CRYOLIB and the correlations of homogeneous equilibrium model are used for the heat transfer and pressure drop in nodes of pipes. The magnet section is separated into the coils and shells. To demonstrate the coils and shells, the cylinder model in the THERMAL library is used. Since the coils are composed of diverse materials, averaged properties are imported to the coils. The outer surfaces of shells of magnet section are connected to cooling pipes by using linear thermal conductors with variable properties. The side surfaces of shells from CS0 to MS2 are thermally connected without thermal resistances.

Table 6 shows all of the values used for the components in the model of EcosimPro. At 
the normal operation, valves (CV172, CV173, CCV1, CCV3, and CCV5) are open and valves (CCV2, CCV4, Recovery on/off valve 1, and Recovery on/off valve 2) are close. Therefore, LHe goes thought the transport magnet first, and then pass the cooling pipe installed on the capture magnet. The supply and return tanks have valves with PID controllers to control the pressure and level of LHe in the tanks. The flow rate of LHe is controlled by using CCV1 and a heater installed in the supply tank. When CS1 quenches, valves (CCV2, CCV5, RV1, and RV2) are open and valves (CV172, CV173, CCV3, and CCV4) are close. Therefore, helium in the transfer tubes, cooling pipes and return pipe is recovered in the buffer tank. All operation time is for $2500 \mathrm{~s}(0-1500 \mathrm{~s}$ : stabilization and normal condition, $1500-1650 \mathrm{~s}$ : the quench happens with the imported heat profile, 1650 - 2500 s: stabilization). When CS1 quenches, we neglect heat generation that would result from discharging the other coils and the transport magnet in the dynamic simulation. The size of communication interval is $0.005 \mathrm{~s}$ to notice very fast transients during the dynamic simulation. 


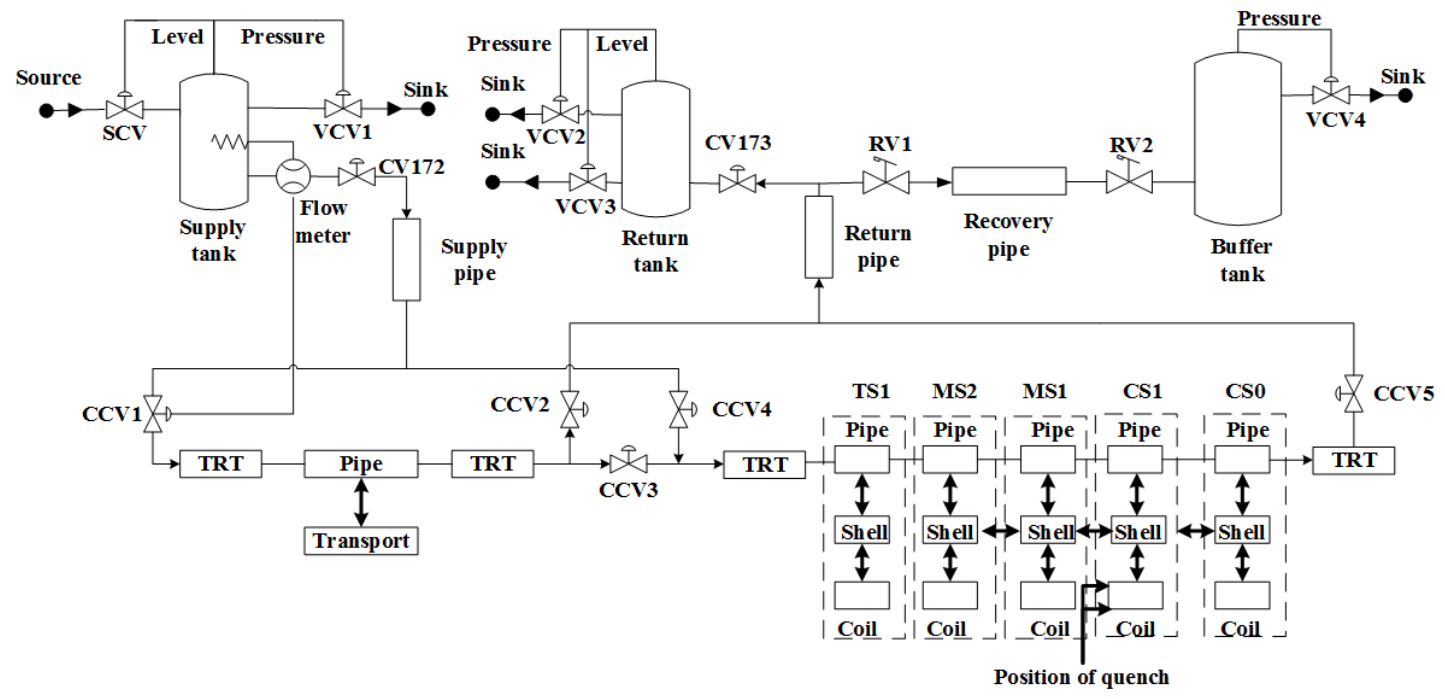

Fig. 9. Developed diagram for dynamic simulation (RV1: Recovery on/off valve 1,

RV2: Recovery on/off valve 2).

\section{Table 6}

Values for components at dynamic simulation.

\begin{tabular}{|c|c|}
\hline Components & Values \\
\hline $\begin{array}{l}\text { Mass flow rate of } \\
\text { LHe }\end{array}$ & $32 \mathrm{~g} / \mathrm{s}$, source of supply tank : $1.41 \mathrm{bar}$ and $4.5 \mathrm{~K}$ \\
\hline $\begin{array}{l}\text { Supply and return } \\
\text { pipes }\end{array}$ & $\begin{array}{l}\text { Material: stainless steel } 316 \text {, outer diameter: } 27.2 \mathrm{~mm} \text {, thickness: } 2.1 \mathrm{~mm} \text {, } \\
\text { length: } 5 \mathrm{~m} \text {, heat load }=12 \mathrm{~W} \text { for each pipe }\end{array}$ \\
\hline $\begin{array}{l}\text { Transfer tubes } \\
\text { (supply and return) }\end{array}$ & $\begin{array}{l}\text { Material: stainless steel } 316 \text {, outer diameter: } 27.2 \mathrm{~mm} \text {, thickness: } 2.1 \mathrm{~mm} \text {, } \\
\text { length : } 10 \mathrm{~m} \text {, heat load }=4 \mathrm{~W} \text { for each pipe }\end{array}$ \\
\hline $\begin{array}{l}\text { Cooling pipe of } \\
\text { capture magnet }\end{array}$ & $\begin{array}{l}\text { Material: aluminum 6061, outer diameter: } 31 \mathrm{~mm} \text {, thickness: } 3 \mathrm{~mm} \text {, length: } 58 \\
\mathrm{~m}(\mathrm{CS} 0: 6 \mathrm{~m}, \mathrm{CS} 1: 14 \mathrm{~m}, \mathrm{MS} 1: 14 \mathrm{~m}, \mathrm{MS} 2: 12 \mathrm{~m}, \mathrm{TS} 1: 12 \mathrm{~m})\end{array}$ \\
\hline $\begin{array}{l}\text { Cooling pipe of } \\
\text { transport magnet }\end{array}$ & $\begin{array}{l}\text { Material: aluminum 6061, outer diameter: } 31 \mathrm{~mm} \text {, thickness: } 3 \mathrm{~mm} \text {, length: } 50 \\
\mathrm{~m}\end{array}$ \\
\hline Recovery pipe & $\begin{array}{l}\text { Material: stainless steel 316, outer diameter: } 76.3 \mathrm{~mm} \text {, thickness: } 3 \mathrm{~mm} \text {, } \\
\text { length: } 70 \mathrm{~m}\end{array}$ \\
\hline Buffer tank & Volume: $20 \mathrm{~m}^{3}$ \\
\hline
\end{tabular}


Material: coil (averaged property), shell (aluminum 5083), inner diameter:

CS0 $1.374 \mathrm{~m}$ (coil) and $1.675 \mathrm{~m}$ (shell), outer diameter: $1.675 \mathrm{~m}$ (coil) and $1.755 \mathrm{~m}$ (shell), length: $0.2 \mathrm{~m}$, total heat load: $19 \mathrm{~W}$

Material: coil (averaged property), shell (aluminum 5083), inner diameter: $1.344 \mathrm{~m}$ (coil) and $1.67 \mathrm{~m}$ (shell), outer diameter: $1.67 \mathrm{~m}$ (coil) and $1.747 \mathrm{~m}$ (shell), length: $1.411 \mathrm{~m}$, total heat load: $136 \mathrm{~W}$

Material: coil (averaged property), shell (aluminum 5083), inner diameter: $1.344 \mathrm{~m}$ (coil) and $1.51 \mathrm{~m}$ (shell), outer diameter: $1.51 \mathrm{~m}$ (coil) and $1.63 \mathrm{~m}$ (shell), length: $1.488 \mathrm{~m}$, total heat load: $59 \mathrm{~W}$

Material: coil (averaged property), shell (aluminum 5083), inner diameter:

MS2 $1.344 \mathrm{~m}$ (coil) and $1.578 \mathrm{~m}$ (shell), outer diameter: $1.578 \mathrm{~m}$ (coil) and 1.692 (shell), length: $0.742 \mathrm{~m}$, total heat load: $26.6 \mathrm{~W}$

Material: coil (averaged property), shell (aluminum 5083), inner diameter: 0.5 - $0.82 \mathrm{~m}$, outer diameter: $0.718-1.1 \mathrm{~m}$, length: $\sim 1.7 \mathrm{~m}$, total heat load: $6.7 \mathrm{~W}$

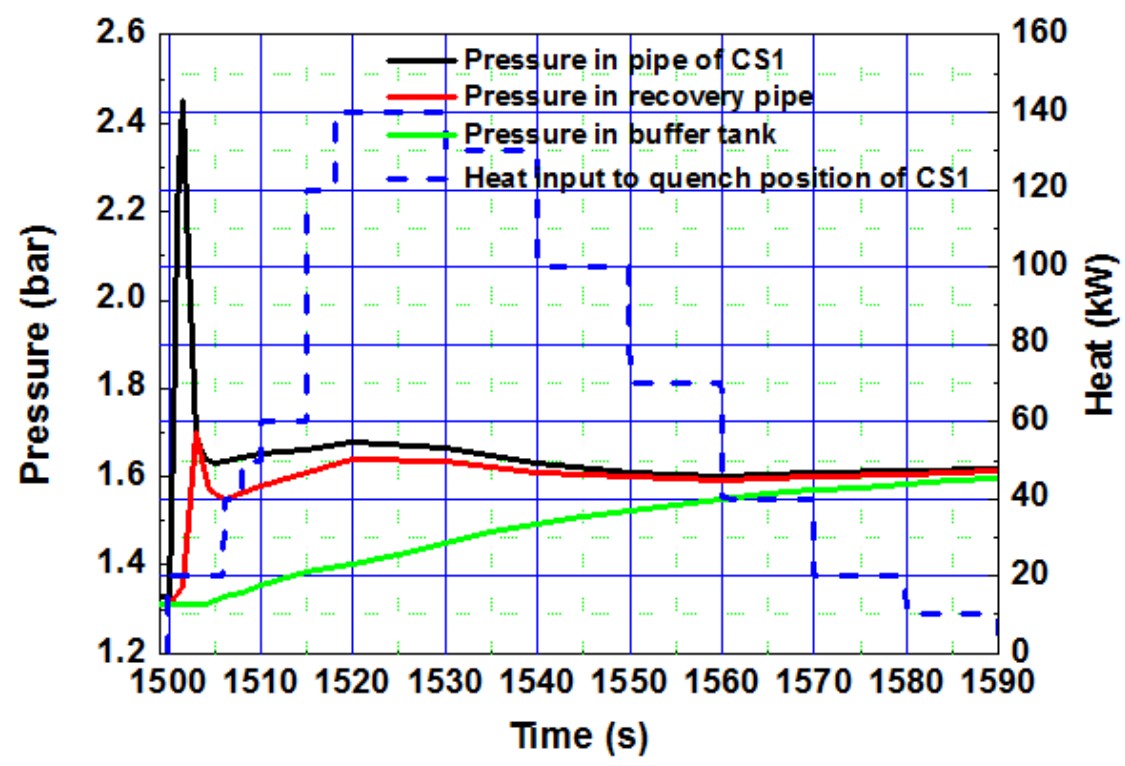

Fig. 10. Pressure and heat curves of second case during quench. 


\subsection{Results}

There are two cases for the dynamic simulation. In the first case, the quench position is the edge of inner surface of CS1 coil and the condition makes CS1 coil have the maximum temperature because the distance between the location of quench and the cooling pipe is the largest. In the second case, the quench position is the edge of outer surface of CS1 coil and the condition makes the cooling pipe have the maximum pressure. Fig. 10 shows the pressure and heat input curves of the second case during the quench. After $1505 \mathrm{~s}$, pressures are averaged because the pressures oscillate by the instability of helium in the cooling pipe of the capture magnet. At the first and second cases, the maximum pressures of the cooling pipe are 1.7 bar and 2.5 bar, respectively. The volume of the recover section (recovery pipe and buffer tank) and the small quantity of liquid helium in the cooling pipe prevent pressure increasing too high when CS1 quenches. Therefore, we can confirm that we do not need to install a relief valve on the magnets. The helium temperature at the inlet port of buffer tank is over $295 \mathrm{~K}$. It means that a heater for increasing the temperature of cold helium gas is not necessary in the recovery pipe. 


\section{Discussion}

In this paper, we explain the current statuses of cryogenic system and superconducting magnets for the COMET phase I and phase II experiments. We still have additional challenges to develop the systems completely. For the capture magnet, we want to use Al strips to reduce the temperature differences between layers of coils. Since it is technically difficult to install perfectly Al strips between layers of coils and we need to develop a stable method. The heat generated from neutrons is obtained from Monte Carlo simulation [10] and the simulation tool is still being updated. The heat at phase II may be changed because other minor components such as supports of cryostat will be included in the simulation tool. But, the cryogenic cooling system will be designed to have enough margins and will be compatible with the changes.

There are three necessary studies for the cryogenic system. One is for the transient simulation to estimate the cool-down and warm-up time. We are developing a simulation model composed of a compressor, a cold box, a current lead box with transfer tubes, a magnet system, and a recovery system in EcosimPro. It is also for checking our operation schemes and PID controls for the cryogenic system. Second one is for the design of transfer tubes. There is no standard design scheme for a transfer tube with 
superconducting lead wires. KEK has some experience with this from having developed the transfer tube with rectangular-shaped superconducting wires for ATLAS [11]. But there are still unknown factors to design the transfer tube. It is required to make a standardized and settled design method. We will publish a paper including all of the works for our transfer tubes. The last one is about updating the previous quench analysis. The quench simulation requires multiphysics. Our previous quench analysis [7] included radiation, electromagnetic, electrical, and thermal effects for CS1. We need to add a fluid dynamic model to consider the heat transfer between the magnet and cooling pipe and include other coils in the previous analysis.

\section{Summary}

Construction of superconducting magnets and an overall cryogenic system for the COMET phase I and phase II experiments is in progress. In this paper, the cryogenic system composed of a cooling structure, a liquefier, a current lead box, transfer tubes is discussed and current status of the superconducting magnets is updated. To satisfy requirements for the experiments of phase I and phase II, a capture solenoid and a transport solenoid were contrived and a half of the transport solenoid is installed at the hadron hall of J-PARC. Design and modification for the capture solenoid, which will be 
at a high irradiation condition, are almost finished. We are focusing on design of the cryogenic system to support stably the operation of the magnets. To cool down the superconducting magnets at the radiation environment, a conduction-cooling method is applied. The required mass flow rates and flow conditions of liquid helium are checked. The estimated total head loads at $4.5 \mathrm{~K}$ systems of phase I and phase II are $67 \mathrm{~W}$ and 295 $\mathrm{W}$, respectively. Our Linde TCF 50 liquefier is compatible with the operation of phase I. But, for the operation of phase II, it is necessary to replace it with a new liquefier which has higher heat capacity and supplies the mass flow rate of liquid helium as large as 32 $33 \mathrm{~g} / \mathrm{s}$ to the magnets. The current lead box and unique transfer tubes are being designed to supply currents and liquid helium to the magnets. Especially, it is important to check the maximum pressure in the cooling pipe which is installed on the magnets, because we do not want to install a relief valve on the magnets due to activated helium. By using dynamic simulation in EcosimPro, we check that the maximum pressure in the cooling pipe is not over 2.5 bar when CS1 of the capture magnet quenches.

\section{Acknowledgments}

This work was supported by Japan Science and Technology Agency under Strategic Promotion of Innovative Research and Development Program (S-Innovation Program) 
and Japan Society for the Promotion of Science Grants-in-Aid for Scientific Research (KAKENHI). And Ki. T was supported by Basic Science Research Program through the National Research Foundation of Korea (NRF) funded by the Ministry of Education, Science, and Technology (2015R1A6A3A03018889).

\section{References}

[1] COMET Collaboration. An experimental Proposal on Nuclear and particle physics experiments at the J-PARC $50 \mathrm{GeV}$ proton synchrotron. Main proposal 2007.

[2] COMET Collaboration. Technical design report 2014.

[3] Yoshida M., Nakamoto T., Ogitsu T., et al. Superconducting solenoid magnets for the COMET experiment. IEEE Trans. Appl. Supercond., 2011, 21(3):1730-1733.

[4] Yoshida M., Yang Y., Ogitsu T., et al. Status of Superconducting solenoid system for COMET phase-I experiment at J-PARC. IEEE Trans. Appl. Supercond., 2015, 25(3):Art. ID. 4500904.

[5] Yoshida M., Nakamoto T., Ogitsu T., et al. Low-temperature neutron irradiation tests of superconducting magnet materials using reactor neutrons at KUR. in Pro. AIP Conf., 2011, 1435:167-173. 
[6] Yoshida M., Mihara S., Nakamoto T., et al. Development of a radiation resistant superconducting solenoid magnet for mu-e conversion experiment. IEEE Trans. Appl. Supercond., 2013, 23(3):Art. ID. 4101404.

[7] Yang Y. Radiation study on the superconducting solenoid magnet for $\mu^{-}->e^{-}$ conversion experiment. Mater degree thesis, Kyushu University (2015).

[8] Haruyama T., Mito T., Doi Y., and Yamamoto A. Pressure drop in forced two phase cooling of the large thin superconducting solenoid. Adv. Cryog. Eng., 1988, 33:543549.

[9] Agrupados E. EcosimPro 5.4: Modelling and simulation software (2015), http:// www.ecosimpro.com.

[10] Sato T., Niita K., Matsuda N., et al. Particle and heavy ion transport code system PHITS - version 2.52. J. Nucl. Sci. Technol., 2013, 50(9):913-923.

[11] Design documentation for ATLAS Magnet System, http://atlasmagnet.web.cern.ch/atlas-magnet/cryo/docs/design. 